\title{
IMAGE DECONVOLUTION OF THE RADIO RING PKS 1830-211
}

\author{
F. Courbin \\ Institut d'Astrophysique et de Géophysique, Université de Liège, Avenue de Cointe 5, B-4000 Liège, Belgium; \\ URA 173 CNRS-DAEC, Observatoire de Paris, France \\ C. LIDMAN \\ European Southern Observatory, Casilla 19001, Santiago 19, Chile \\ B. L. FRYE \\ Astronomy Department, University of California, Berkeley, Berkeley, CA 94720 \\ P. MAGAIN ${ }^{2}$ \\ Institut d'Astrophysique et de Géophysique, Université de Liège, Avenue de Cointe 5, B-4000 Liège, Belgium \\ T. J. BROADHURST \\ Astronomy Department, University of California, Berkeley, Berkeley, CA 94720 \\ AND \\ M. A. PAhre ${ }^{3,4}$ AND S. G. DJorgovsKi \\ Palomar Observatory, California Institute of Technology, Mail Stop 105-24, Pasadena, CA 91125 \\ Received 1998 February 12; accepted 1998 April 2; published 1998 May 6
}

\begin{abstract}
New high-quality Keck and ESO images of PKS 1830-211 are presented. By applying a powerful new deconvolution algorithm to these optical and infrared data, both images of the flat spectrum core of the radio source have been identified. An extended source is also detected in the optical images which is consistent with the expected location of the lensing galaxy. The source counterparts are very red at $I-K \sim 7$, which suggests strong Galactic absorption with additional absorption by the lensing galaxy at $z=0.885$ and is consistent with the detection of high-redshift molecules in the lens.
\end{abstract}

Subject headings: cosmology: observations — gravitational lensing — infrared: general — methods: data analysis - quasars: individual (PKS 1830-211) techniques: image processing

\section{INTRODUCTION}

The bright radio source PKS 1830-211 (Subrahmanyan et al. 1990, hereafter S90; Jauncey et al. 1991) has attracted much attention as the most detailed example of a lensed radio ring. Among the classically lensed QSOs, its short time delay of 44 days (van Ommen et al. 1995) and its clean lens geometry make it a good candidate for measuring $H_{0}$. The lens, a gasrich galaxy at $z=0.89$, was discovered in the millimeter via molecular absorption (Wiklind \& Combes 1996), which is seen toward only one of the two flat-spectrum hot spots (Wiklind \& Combes 1996; Frye, Welch, \& Broadhurst 1997). The presence of a nearby $\mathrm{M}$ star as well as heavy extinction along the line of sight $\left(b=-5^{\circ} .7\right)$ had until now hampered the identification of the lens and the source. In this Letter, we describe how the MCS deconvolution algorithm (Magain, Courbin, \& Sohy 1998) was used to detect the counterparts of this bright radio ring in deep Keck optical and infrared images.

\section{OBSERVATIONS AND REDUCTIONS}

Near-IR $J\left(\lambda_{c}=1.25 \mu \mathrm{m}\right)$ and $K^{\prime}\left(\lambda_{c}=2.15 \mu \mathrm{m}\right)$ images were obtained on the nights of 1997 April 14 and 15 with the IRAC2b camera on the ESO/MPI $2.2 \mathrm{~m}$ telescope, which uses

\footnotetext{
${ }^{1}$ Based on observations obtained at the ESO La Silla Observatory, Chile and at the W. M. Keck Observatory, Hawaii, which is operated jointly by the California Institute of Technology and the University of California.

${ }^{2}$ Also at Maître de Recherches au FNRS (Belgium).

${ }^{3}$ Present address: Harvard-Smithsonian Center for Astrophysics, 60 Garden Street, Mail Stop 20, Cambridge, MA 02140.

${ }^{4}$ Hubble Fellow.
}

a NICMOS3 $256 \times 256 \mathrm{HgCdTe}$ array. The good seeing $(0,6-0.7)$ and the fact that a good sampling is desirable for deconvolution led us to choose the smallest pixel size available on this instrument, i.e., 0 ". 151 , which resulted in a total field of view of $38^{\prime \prime}$ on a side. The data were taken and reduced exactly in the same way as in Courbin, Lidman, \& Magain (1998). Several standard stars were observed during the night. The standard deviation in the zero points was less than 0.02 mag for both $J$ and $K^{\prime}$. The IR magnitudes reported in this paper are on the $J H K$ system of Bessell \& Brett (1988).

Near-IR Keck I data were obtained on the night of 1994 April 5 with the Near-IR Camera (NIRC) (Matthews \& Soifer 1994). Five $80 \mathrm{~s} K$ images were combined using the sigma clipping algorithm available in IRAF. The pixel size is 0".157, which is similar to that used with IRAC2b. The images were obtained under marginally nonphotometric conditions, with a zero point uncertain by about $0.1 \mathrm{mag}$. Due to the crowding of the field and the low number of frames available, sky subtraction was imperfect but did not influence dramatically the quality of the data analysis.

Six dithered $I$-band images were obtained during the full moon night of 1997 June 15 using the Keck II telescope and the LRIS (Oke et al. 1995). The CCD detector was a Tektronix $2048 \times 2048$ with a pixel size of $0{ }^{\prime \prime} 215$. The individual exposure times were restricted to 3 minutes in order to avoid saturation of the brightest stars in this extremely crowded field. The images were bias-subtracted and flat-fielded in the standard way. All of them have a seeing close to 0 " 8 . No standard star was observed, but a flux calibration could be performed relative to an $I$-band image taken at La Silla with the $0.9 \mathrm{~m}$ telescope on the night of 1997 April 15. 

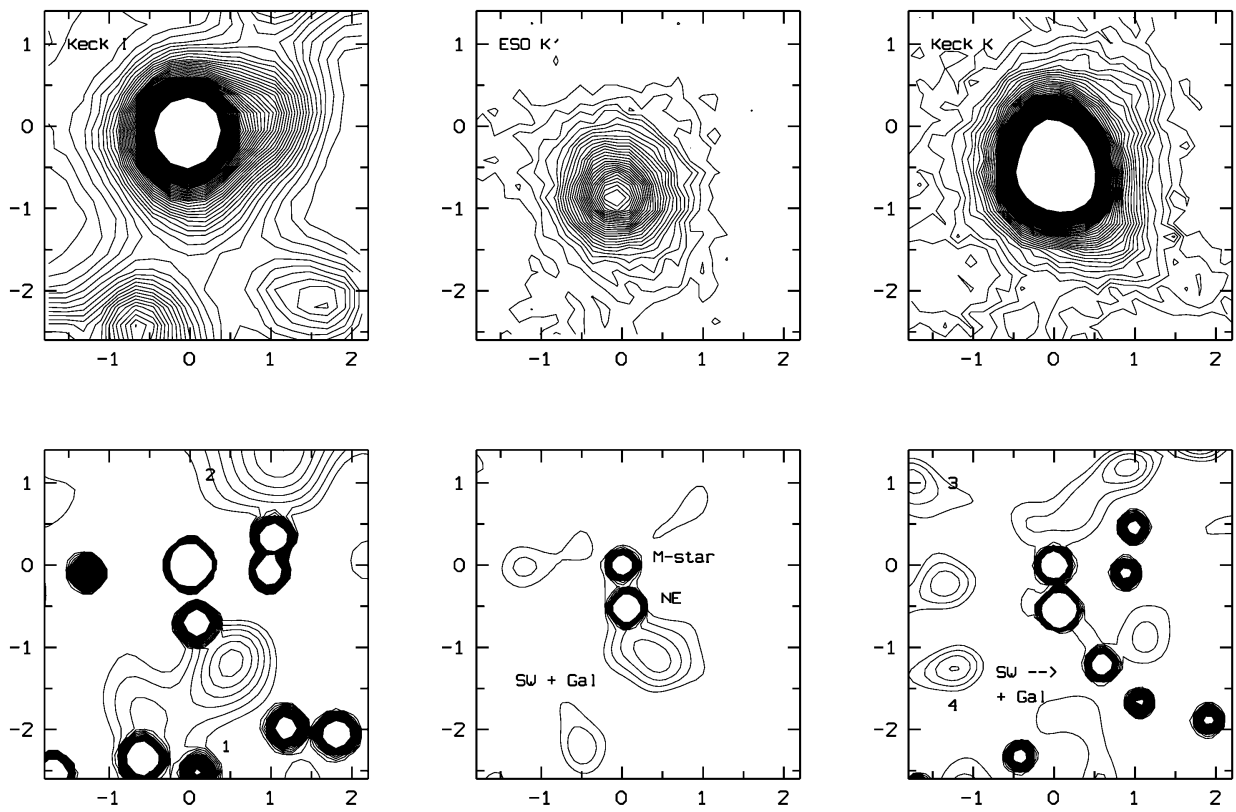

FIG. 1.-The 4" field around PKS 1830-211 observed in the $I, K^{\prime}$, and $K$ bands at Keck and ESO observatories. Top left: Stack of six $I$-band frames with a pixel size of 0.215 and seeing of $0{ }^{\prime \prime} 8$. Top middle: Mean of four temporary stacks (see text) obtained in the $K^{\prime}$ band with the ESO/MPI $2.2 \mathrm{~m}$ telescope. The pixel size is 0.151, and the seeing is 0.7. Top right: Mean of five $K$-band images obtained with Keck I and NIRC. The pixel size is 0 ". 157 , and the seeing is 0.7. Bottom right: Simultaneous deconvolution of the six I-band frames: resolution of 0".215 and pixel size of 0"1075. Bottom middle: Simultaneous deconvolution of the four $K^{\prime}$ images: final resolution of 0.151 and pixel size of 0.075 . Bottom right: Deconvolution of the mean of five NIRC images: resolution of 0"157 and pixel size of 0"0785. In all of the images, north is up, and east is to the left. The M star, the northeast QSO component candidate, and the blended southwest component + lensing galaxy candidate are indicated. In all images the first contour corresponds to $2.5 \sigma_{\text {sky. }}$ The step between two contours is $2.5 \sigma_{\text {sky }}$.

\section{IMAGE DECONVOLUTION}

The MCS deconvolution code (Magain et al. 1998) was applied to all of the images. Due to the low signal-to-noise ratio $(\mathrm{S} / \mathrm{N})$ and the numerous bad pixels in single IR images, these were medianed in groups of nine dithered and sky-subtracted frames. The resulting images have better $\mathrm{S} / \mathrm{N}$ and cosmetics. Two infrared-bright nearby stars, although outside the field of view, produced scattered light in the field, forcing us to reject a fair fraction $(\sim 40 \%)$ of our observations in the $K^{\prime}$ band. One of the culprits was the known source IRAS 18306-2106. Two stacks were obtained in $J$ (total exposure time of $1920 \mathrm{~s}$ ) and four in $K^{\prime}$ (total exposure time of $1440 \mathrm{~s}$ ). Only one such stack was obtained for the IR Keck images, since we had only five dithered frames to combine (total exposure time equal to 400 s).

\subsection{Application of the MCS Code to the Present Data}

The deconvolution process is the same as in Courbin et al. (1998). We chose a pixel scale in the deconvolved images that is a factor of 2 smaller than the pixel scale in the original data to ensure that the sampling theorem is satisfied in the decon-

TABLE 1

Summary of the Photometry

\begin{tabular}{ccccc}
\hline \hline Band & M Star & $\begin{array}{c}\text { Northeast } \\
\text { Component }\end{array}$ & $\begin{array}{c}\text { Southwest } \\
\text { Component }+ \text { Lens }\end{array}$ & $\begin{array}{c}\text { Limiting } \\
\text { Magnitude }\end{array}$ \\
\hline$I$ (Keck) $\ldots \ldots \ldots$ & $19.3 \pm 0.1$ & $22.0 \pm 0.2$ & $22.3 \pm 0.3$ & 24.0 \\
$J$ (ESO) $\ldots \ldots .$. & $18.1 \pm 0.1$ & $18.7 \pm 0.3$ & $>20.5$ & 20.5 \\
$K^{\prime}$ (ESO) $\ldots \ldots$ & $17.3 \pm 0.3$ & $15.8 \pm 0.2$ & $19.0 \pm 0.4$ & 19.1 \\
$K$ (Keck) $\ldots \ldots$ & $16.6 \pm 0.2$ & $15.1 \pm 0.1$ & $18.2 \pm 0.2$ & 21.3 \\
\hline
\end{tabular}

Note. - Results are given for the $\mathrm{M}$ star, the northeast component, and the southwest component of the lensed source plus lensing galaxy. The limiting magnitude (point sources) for each of the bands is given in the final column. volved images. After deconvolution, the resolution is fixed to 2 pixels FWHM for all data. The corresponding final pixel scales and resolutions on the plane of the sky are given in Figure 1.

We constructed the infrared point-spread function (PSF) from the relatively isolated, bright star labeled $\mathrm{H}$ in Djorgovski et al. (1992, hereafter D92). In the optical images, the stars mentioned in D92 are all saturated. Consequently, the PSF was constructed from four fainter stars about $30^{\prime \prime}$ away from the radio ring. Crowding in the optical field made the quality of the PSF rather poor, especially in the outer wings of the hexagonal Keck PSF, which introduced systematic residuals into the deconvolved image and residual maps (see $\S 3.2$ ).

In each band, all of the frames available were deconvolved simultaneously, i.e., six in $I$, two in $J$, four in $K^{\prime}$, and one in $K$. In other words, the output of the procedure is a unique deconvolved image that is simultaneously compatible with all of the images included in the data set. The deconvolved image is given as a sum of point sources with known Gaussian shape and a diffuse background smoothed on the length scale of the final resolution chosen by the user. The photometry and the astrometry of the point sources are also obtained as by-products of the deconvolution and are provided in Tables 1 and 2 .

\subsection{Quality Check of the Deconvolution}

Many deconvolution algorithms generate the so-called deconvolution artifacts as well as noise enhancement. Even if the MCS code does not produce artifacts, it still has to accomplish the difficult task of deconvolving the image from imperfect and noisy data. An objective criterion has therefore been established to check the quality of the results. A natural product of the MCS code is the residual map (RM), which is the difference between the deconvolved and real images divided by 
TABLE 2

SUMMARY OF THE ASTROMETRY

\begin{tabular}{ccc}
\hline \hline Parameter & $\begin{array}{c}\text { Northeast } \\
\text { Component }\end{array}$ & $\begin{array}{c}\text { Southwest } \\
\text { Component }+ \text { Lens }\end{array}$ \\
\hline$x(I) \ldots \ldots \ldots$ & $+0.08 \pm 0.1$ & $+0.48 \pm 0.1$ \\
$y(I) \ldots \ldots \ldots$ & $-0.70 \pm 0.1$ & $-1.15 \pm 0.1$ \\
$x(J) \ldots \ldots \ldots$ & $-0.07 \pm 0.1$ & $\cdots$ \\
$y(J) \ldots \ldots$. & $+0.54 \pm 0.1$ & $\cdots$ \\
$x\left(K^{\prime}\right) \ldots \ldots$ & $+0.06 \pm 0.07$ & $+0.54 \pm 0.07$ \\
$y\left(K^{\prime}\right) \ldots \ldots$. & $-0.52 \pm 0.07$ & $-1.16 \pm 0.07$ \\
$x(K) \ldots \ldots$. & $+0.06 \pm 0.05$ & $+0.59 \pm 0.05$ \\
$y(K) \ldots \ldots$. & $-0.54 \pm 0.05$ & $-1.20 \pm 0.05$ \\
\hline
\end{tabular}

Note. - Results for the northeast and southwest components of the lensed source plus lensing galaxy are given in arcseconds, relative to the $\mathrm{M}$ star, together with their $1 \sigma$ error bars.

the standard deviation of each pixel. Whenever the simultaneous deconvolution capability of the method is used, as in the present case, the program returns one RM for each frame in the data set. A perfect deconvolved image should match the data at best in the sense of the $\chi^{2}$ and leave a flat RM with a mean value of $1(1 \sigma)$ all over the field.

RMs guide the user in his/her choice of the smoothing to be applied to the image in order to avoid local under- or overfitting of the data. Still, on the basis of the RMs, the user can constrain the number of point sources to be involved in the deconvolution. For example, missing a bright point source (i.e., one with a high $\mathrm{S} / \mathrm{N}$ ) will result in a "hot spot" well above the critical value of 1 in the RMs. This is no longer true for very faint sources. In this case, no discrimination can be made between an extended object and a point source, so the faint point sources are modeled as part of the deconvolved background. We therefore always choose the minimum number of point sources needed in order to produce acceptable RMs. Thus, different images of the same field can have different aspects, depending on whether individual objects were modeled as point sources or not.

Objects near the frame edges are not well fitted, especially in crowded fields such as these (e.g., objects labeled 1-4 in Fig. 1). Object 1 leaves particularly significant structures in the RMs of the $I$-band data, either when modeled as a point source or as part of the background in the deconvolved image. We therefore conclude that it is extended or that it is a very strong blend of point sources.

In order to run the MCS algorithm, the user has to provide initial positions and intensities for all point sources. The astrometric $1 \sigma$ error bars were estimated as the dispersion of the results of several deconvolutions using different initial conditions. The typical astrometric accuracy is $00^{\prime \prime} 05$ for the brightest point sources, while it can be as much as 0.2 for the faintest ones. Since the peak intensity of a point source is allowed to be different in each data frame, the photometric error bar is simply the dispersion of the peak intensities found after simultaneous deconvolution of a whole data set. In order to quantify the additional errors introduced by a poor knowledge of the PSF in the Keck $I$ band, the deconvolution program was run using five different PSFs computed from different stars in the field.

\section{RESULTS AND DISCUSSION}

In Figures 1 and 2, we present both the raw and deconvolved images in $I, K^{\prime}$, and $K$, with a resolution of the order of 0 ".15-0.20. A red point source is clearly detected at the position

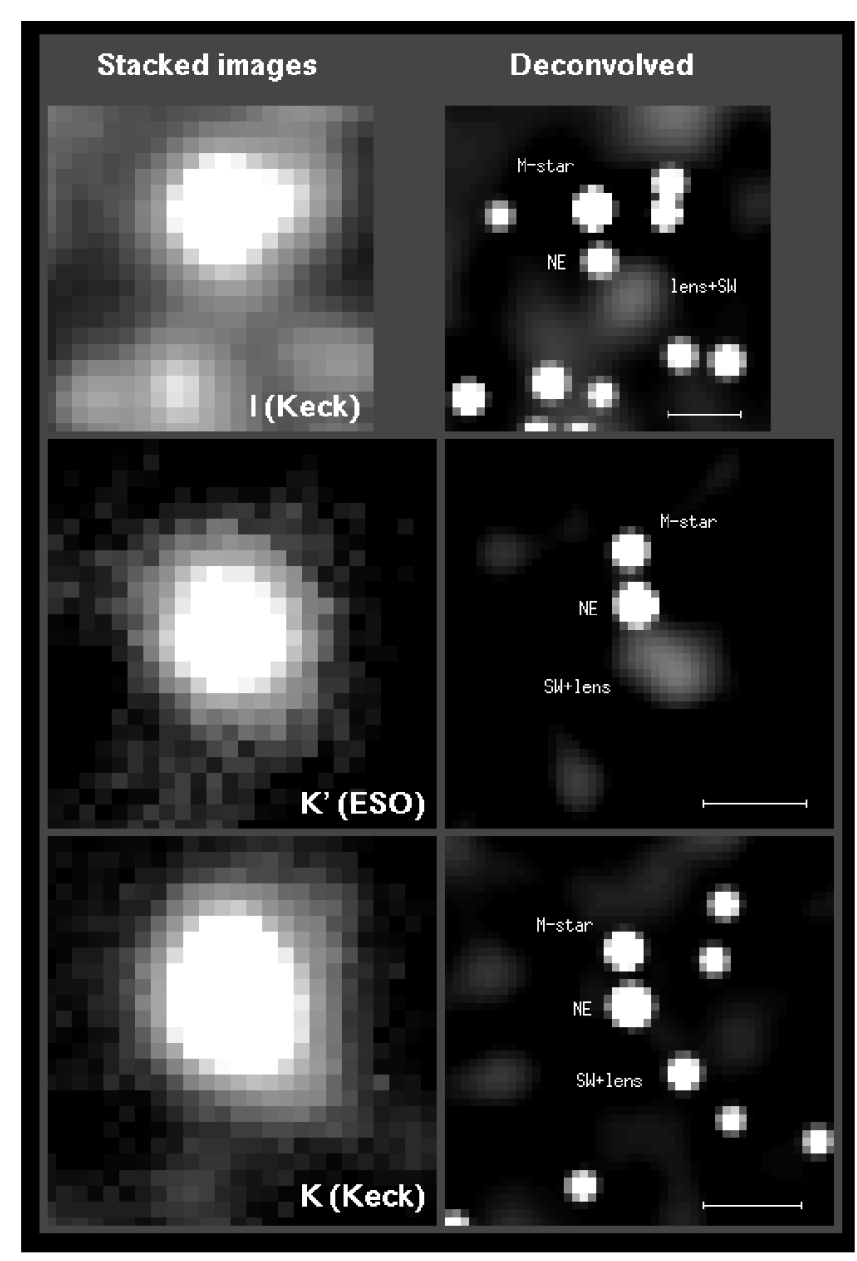

FIG. 2.-Field around PKS 1830-211 observed in the $I, K^{\prime}$, and $K$ bands at the Keck and ESO observatories. Top left: Stack of six I-band frames with a pixel size of 0.215 and seeing of $0{ }^{\prime \prime} 8$. Middle left: Mean of four temporary stacks (see text) obtained in the $K^{\prime}$ band with the ESO/MPI $2.2 \mathrm{~m}$ telescope. The pixel size is 0.151 and the seeing is 0"7. Bottom left: Mean of five $K$ band images obtained with Keck I and NIRC. The pixel size is 0.157 , and the seeing is 0"7. Top right: Simultaneous deconvolution of the six $I$-band frames: resolution of 0"215 and pixel size of 0"1075. Middle right: Simultaneous deconvolution of the $4 K^{\prime}$ images: final resolution of 0.151 and pixel size of 0"075. Bottom right: Deconvolution of the mean of five NIRC images: resolution of $00^{\prime \prime} 157$ and pixel size of $00^{\prime \prime} 0785$. In all of the images north is up, and east is to the left. The M star, the northeast QSO component candidate, and the blended southwest component + lensing galaxy candidate are indicated. The horizontal line in each deconvolved image is 1 " long. The cuts of the images are chosen in order to display the full dynamics of the image at low light levels.

expected for the northeast radio source of PKS 1830-211. Another red object is observed close to the position of the southwest radio source of the lensed system, but the extended nature of the source and the poor quality of the PSF do not allow us to sort out its detailed morphology. The photometry and astrometry of the field are presented in Tables 1 and 2 and in Figure 3, along with our estimates of the $1 \sigma$ error bars.

The red star in D92 dominates the total flux in the $I$ band, while in the infrared, the component near the northeast radio source takes over. With our high $\mathrm{S} / \mathrm{N}$ we can show that its shape is compatible with a point source, and its color, $I-$ $K=6.9$, is much redder than any "normal" star (e.g., Koornneef 1983). The $I, K^{\prime}$, and $K$ positions are all within the $1 \sigma$ radio error bars. In Figure 3, all objects are aligned with respect to the $\mathrm{M}$ star so that we can compare the different positions 


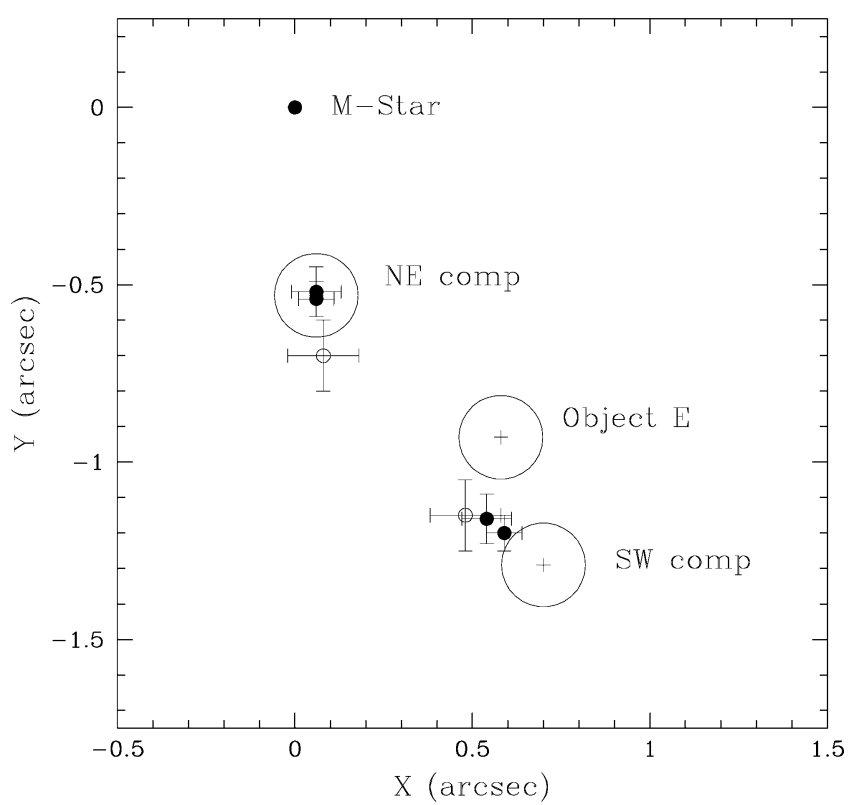

FIG. 3.-Positions observed for the different objects detected in the optical and near-IR along with their $1 \sigma$ error bars relative to the $\mathrm{M}$ star. The large open circles are radio positions whose radius corresponds to the error bars quoted by $\mathrm{S} 90$. The filled circles show the positions measured from the nearIR images, while the open circles show the result obtained from the $I$-band data.

measured for the northeast and southwest components at optical and IR wavelengths.

Molecular absorption toward the two lensed images was spatially resolved at millimeter wavelengths, and the separation between the lensed images was found to be 0.98 (Frye et al. 1997). In our optical and IR images, the southwest component is $0.61 \pm 0.13$ and $0.85 \pm 0.09^{\prime \prime}$, respectively, away from the northeast component. A plausible explanation for the apparent positional shift between the optical, IR, and radio positions is that the southwest component is a blend of two objects: the lensing galaxy and the heavily reddened southwest component seen in the radio images. This has been very recently confirmed by Hubble Space Telescope (HST) NICMOS3 observations by the CASTLES group (McLeod al. 1998) which show the lensing galaxy in the center of the "hole" of the radio ring, as predicted by LensClean modeling of the system by Kochanek $\&$ Narayan (1992). Note also the very good general agreement between the HST images and our deconvolutions, although the latter were carried out without prior knowledge of the HST data.

The flux ratio between the two lensed images of the source is $0.973 \pm 0.016$ at radio wavelengths (S90) and about 18.2 in $K(3.15 \pm 0.2 \mathrm{mag})$. The combination of a reddened southwest radio source plus blue lens can explain the large flux ratios. Both the northeast and the southwest components are reddened, making PKS 1830-211 another good example of a dusty lens along with MG 0414+0534 (e.g., Annis \& Lupino 1993) and MG 1131+0456 (Larkin, Matthews, \& Lawrence 1994), the mean galactic extinction being far below the values obtained for PKS 1830-211. At the lens redshift, the observed
$K$ band corresponds to a central wavelength of $1.17 \mu \mathrm{m}$ in the rest frame. For the galactic reddening curve, the extinction in this band is $A_{(1.2 \mu)}=0.37 A_{V}$ (Savage \& Mathis 1981). Thus, the differential extinction is about $8.5 \mathrm{mag}$ in the $V$ band and implies $E(B-V)=2.75 \pm 0.2$, which is in good agreement with the value of 2.4 independently derived from the HST observations (McLeod et al. 1998).

If the total extinction is comparable to the differential extinction between the two images, then the source is attenuated in the observed $K$ band by about $3 \mathrm{mag}$. On the other hand, considering simple SIS or point-mass models, the magnification by the lens may be similar, or somewhat less-maybe 2 mag. So, a reasonable value for the unobscured unlensed $K$ magnitude of the source may be around 14. A typical quasar has $V-K \sim 3$. So the unobscured $V$ magnitude of the source may be about 17, which is bright but not unreasonable for $z \sim 1-$ 2.

\section{CONCLUSION}

The main results of our study are the detection of the optical and near-IR counterpart to the northeast radio source of PKS 1830-211 and the possible detection of the southwest component and lensing galaxy. However, our southwest component candidate might be the lensing galaxy alone or, given the crowding in the field, a red galactic object almost coincident with the position of the southwest radio source. The hypothesis of a demagnified third image of the source (S90) between the two main lensed images is unlikely, since in such a case extinction of the lens would have made it visible in the IR. Furthermore, the IR centroid of the southwest component would have been shifted toward object $E$ rather than toward the radio position of the southwest component.

The higher contrast between the northeast component and nearby $M$ star in the infrared makes near-IR spectroscopy necessary for finding the source redshift. Deep, high-resolution near-IR imaging is needed to reveal the exact nature of the faint southwest component. However, even at the highest resolution attainable, which is $0.2-0.3$ in the IR with HST (in particular in $K$ where the southwest component of PKS $1830-211$ is best visible), deconvolution will be essential to discriminate between the southwest component candidate, the lensing galaxy, and additional faint galactic stars.

We thank Jack Welch, Hy Spinrad, Jens Hjorth, Andreas Jaunsen, Chris Kochanek, and the anonymous referee for useful discussions and comments about the first version of the manuscript. We also thank Alfred Rosenberg who provided the ESO $I$-band frame used for the photometric calibration. The expert help of the staff at ESO and WMKO during the observing runs was very much appreciated. F. C. is supported by contracts ARC 94/99-178 “Action de Recherche Concertée de la Communauté Française" and Pôle d'Attraction Interuniversitaire P4/ 05 (SSTC, Belgium). S. G. D. and M. A. P. acknowledge support from the NSF PYI award AST-9157412. Additional support for M. A. P. was provided by NASA through grant number HF-01099.01-97A from the Space Telescope Science Institute, which is operated by AURA under NASA contract NAS5-26555.

\section{REFERENCES}

Annis, J. E., \& Luppino, G. 1993, ApJ, 407, L69

Bessell, M. S., \& Brett, J. M. 1988, PASP, 100, 1134

Courbin, F., Lidman, C., \& Magain, P. 1998, A\&A, 330, 57

Djorgovski, S. G., et al. 1992, MNRAS, 257, 240 (D92)
Frye, B. L., Welch, W. J. W., \& Broadhurst, T. J. 1997, ApJ, 478, L25

Jauncey, D. L., et al. 1991, Nature, 352, 132

Kochanek, C. S., \& Narayan, R. 1992, ApJ, 401, 461

Koornneef, J. 1983, A\&A, 128, 84 
Larkin, J. E., Matthews, K., \& Lawrence, C. R. 1994, ApJ, 420, L9

Magain, P., Courbin, F., \& Sohy, S. 1998, ApJ, 494, 472

Matthews, K., \& Soifer, B. T. 1994, in Infrared Astronomy with Arrays, ed. I. McLean (Dordrecht: Kluwer), 239

McLeod, B. A., Falco, E. E., Impey, C. D., Kochanek, C. S., Lehar, J., Rix, H. W., Keeton, C. R., \& Peng, C. Y. 1998, in preparation Oke, J. B., et al. 1995, PASP, 107, 375
Savage, B., \& Mathis, J. S. 1981, ARA\&A, 17, 73

Subrahmanyan, R., Narasimha, D., Rao, P., \& Swarup, G. 1990, MNRAS, 246, 263 (S90)

van Ommen, T. D., Jones, D. L., Preston, R. A., \& Jauncey, D. L. 1995, ApJ, 444,561

Wiklind, T., \& Combes, F. 1996, Nature, 379, 139 